\title{
DDIT3/CHOP and the sarcoma fusion oncoprotein FUS-DDIT3/TLS-CHOP bind cyclin-dependent kinase 2 Christoffer Bento ${ }^{\dagger}$, Mattias K Andersson ${ }^{\dagger}$ and Pierre Åman*
}

Address: Lundberg Laboratory for Cancer Research, Department of Pathology, University of Gothenburg, Gothenburg, Sweden

Email: Christoffer Bento - christoffer.bento@llcr.med.gu.se; Mattias K Andersson - mattias.andersson@llcr.med.gu.se;

Pierre Åman* - pierre.aman@llcr.med.gu.se

* Corresponding author †Equal contributors

Published: 17 December 2009

BMC Cell Biology 2009, 10:89 doi:10.1 I86/147I-2121-10-89

Received: I July 2009

Accepted: 17 December 2009

This article is available from: http://www.biomedcentral.com/l47|-2/2I//0/89

(c) 2009 Bento et al; licensee BioMed Central Ltd.

This is an Open Access article distributed under the terms of the Creative Commons Attribution License (http://creativecommons.org/licenses/by/2.0), which permits unrestricted use, distribution, and reproduction in any medium, provided the original work is properly cited.

\begin{abstract}
Background: The DDIT3 gene encodes a transcription factor belonging to the CCAAT/enhancer binding protein (C/EBP) family. It is normally expressed at very low levels but is activated by cellular stress conditions and induces GI arrest and, in some cell types, apoptosis. DDIT3 is found as a part of the fusion oncogene FUS-DDIT3 that is causal for the development of myxoid/round-cell liposarcomas (MLS/RCLS).
\end{abstract}

Results: In the present study, we searched for putative interaction partners of DDIT3 and the oncogenic FUS-DDIT3 among GI cyclins and cyclin-dependent kinases. We found that FUS-DDIT3 and the normal DDIT3 bind CDK2. In addition, CDK2 showed an increased affinity for cytoskeletal proteins in cells expressing FUS-DDIT3 and DDIT3.

Conclusions: We conclude that DDIT3 binds CDK2 and that many of the observed biological effects of DDIT3 may involve interaction with CDK2.

\section{Background}

DDIT3 (GADD153, CHOP) encodes a transcription factor belonging to the CCAAT/enhancer binding protein $(\mathrm{C} /$ EBP) family [1]. DDIT3 is normally transcribed at very low levels but is elevated upon DNA damage in cellular stress conditions [2-6]. The DDIT3 protein has a central role in endoplasmatic reticulum stress and DNA damage response by inducing cell cycle arrest and apoptosis $[7,8]$. DDIT3 has recently been implicated in the stress response leading to death of pancreatic insulin producing $\beta$-cells [9] and it may also be a part of cellular stress conditions causing neurodegenerative disorders [10]. In addition, DDIT3 is believed to be involved in growth cessation and terminal differentiation of lipoblasts, osteoblasts and erythrocytes [11-13]. DDIT3 forms heterodimers with several other C/EBP family members [14] as well as other leu- cine zipper carrying proteins $[15,16]$ and the heterodimers are believed to act as dominant negative inhibitors of transcription [14].

DDIT3 is also a part of a fusion oncogene critical for the development of myxoid/round cell liposarcoma (MLS/ RCLS) $[17,18]$. The tumor cells carry the chromosomal translocation $\mathrm{t}(12 ; 16)(\mathrm{q} 13 ; \mathrm{p} 11)$ that results in fusion of DDIT3 to FUS (also called TLS) or more rarely the $\mathrm{t}(12 ; 22)(\mathrm{q} 13 ; \mathrm{q} 12)$ that fuses DDIT3 to EWSR1 [19-21]. The chimerical FUS-DDIT3 oncoprotein functions as an abnormal transcription factor [22] and localizes to distinct nuclear structures in cultured cells [23,24]. MLS/ RCLS cells exhibit abnormal expression profiles of cell cycle controlling factors and among them cyclins and cyclin-dependent kinases (CDKs) [25]. In addition, the 
DDIT3-binding C/EBP $\alpha$ has been shown to interact and inhibit the kinase activity of CDK2 and CDK4 [26]. Based on these observations, we searched for putative interaction partners of the oncogenic FUS-DDIT3 with G1 cyclins and CDKs as these might provide insight into the molecular mechanisms by which the chimerical oncoprotein induces malignancy.

\section{Results \\ CDK2 and cyclin E colocalize with FUS-DDIT3}

HT1080 cells were transiently transfected with a FUSDDIT3-GFP construct and colocalization between the ectopically expressed fusion oncoprotein and endogenous cyclin D1, cyclin E, CDK2 and CDK4 was investigated by immunofluorescence. Cyclin E and CDK2 showed prominent colocalization with the FUS-DDIT3 protein and were detected in FUS-DDIT3 containing granules in the majority of cells (Figure 1). We found no signs of colocalization between FUS-DDIT3 and CDK4 (Figure 1) or cyclin D1 (not shown). Cells transfected with the empty GFP vector showed no granules and a smooth nuclear distribution of CDK2 and cyclin E (Figure 1).

\section{FUS-DDIT3 binds CDK2 through its DDIT3 part}

Cells were transfected with FUS-DDIT3-GFP and cellular proteins were immunoprecipitated with GFP antibodies and further analyzed by western blot. In pilot experiments, we used HT1080 cells stably expressing FUSDDIT3-GFP and could weakly detect endogenous CDK2 in immunoprecipitates while cyclin $\mathrm{E}$ was not found (data not shown). To further confirm a possible interaction between FUS-DDIT3 and CDK2, we instead transiently transfected cells using cloned CDK2 cDNA expressed in frame with the fluorescent protein DsRed1. Cells co-transfected with CDK2-DsRed1 and either of FUS-DDIT3 or DDIT3 expressing constructs showed presence of CDK2DsRed1 in anti-GFP immunoprecipitates from both FUSDDIT3 and DDIT3 transfected cells (Figure 2a). In contrast, CDK2-DsRed1 was not found in immunoprecipitates of cells transfected with the N-terminal part of FUS present in the FUS-DDIT3 protein or in GFP-transfected control cells (Figure 2a). In a reverse experiment, HT1080 cells were transfected with FUS-DDIT3, FUS-DDIT3 LZ, DDIT3 and GFP constructs and cellular proteins in extracts were immunoprecipitated with anti CDK2 antibodies (Figure 2b). We found that endogenous CDK2 coimmunoprecipitated with DDIT3, FUS-DDIT3 and FUSDDIT3 lacking a leucine zipper domain. These results indicate that the region binding CDK2 is located N-terminally of the leucine zipper part of DDIT3. ClustalW alignment performed between DDIT3 and the related C/EBP $\alpha$ showed conservation of several amino acids in distinct regions shared by the two proteins but that DDIT3 lacks a region similar to the one that binds CDK2 in C/EBP $\alpha$ [26] (Figure 2c).
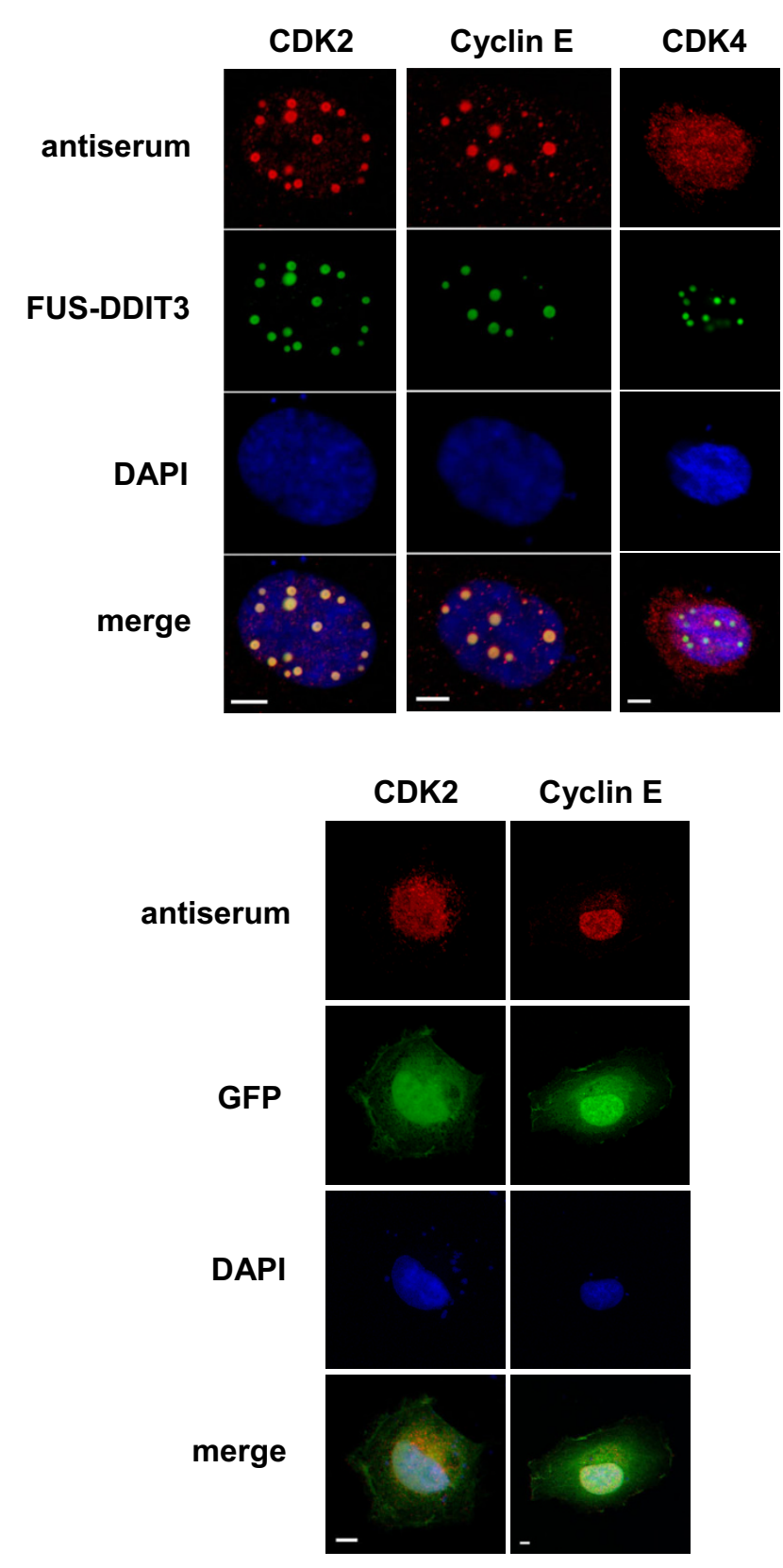

Figure I

CDK2 and cyclin E colocalize with FUS-DDIT3 in transiently transfected HTI 080 cells. The endogenous distribution of CDK2 and cyclin E seen in red is detected by antiserum specific for these proteins. Ectopically expressed GFP-tagged FUS-DDIT3 is shown in green. The DAPI dye is used to stain nuclei blue. CDK4 was not seen to accumulate in FUS-DDIT3-containing granules and no granules were formed in cells overexpressing the GFP protein only. Bars indicate $5 \mu \mathrm{m}$. 
(a)

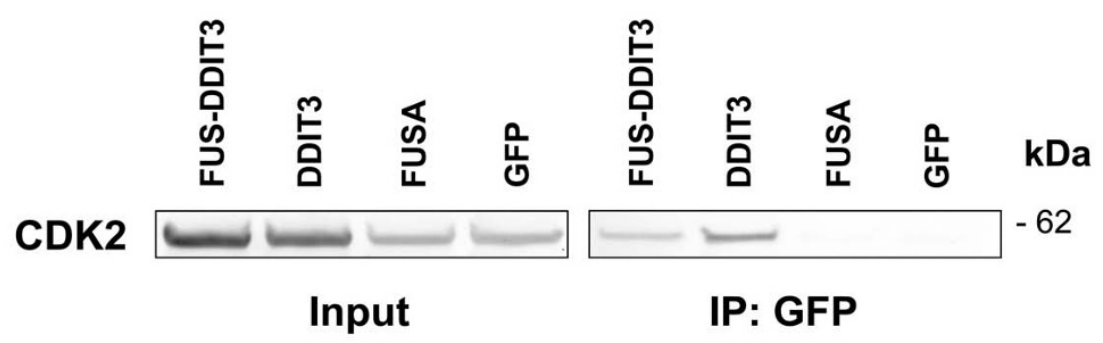

(b)
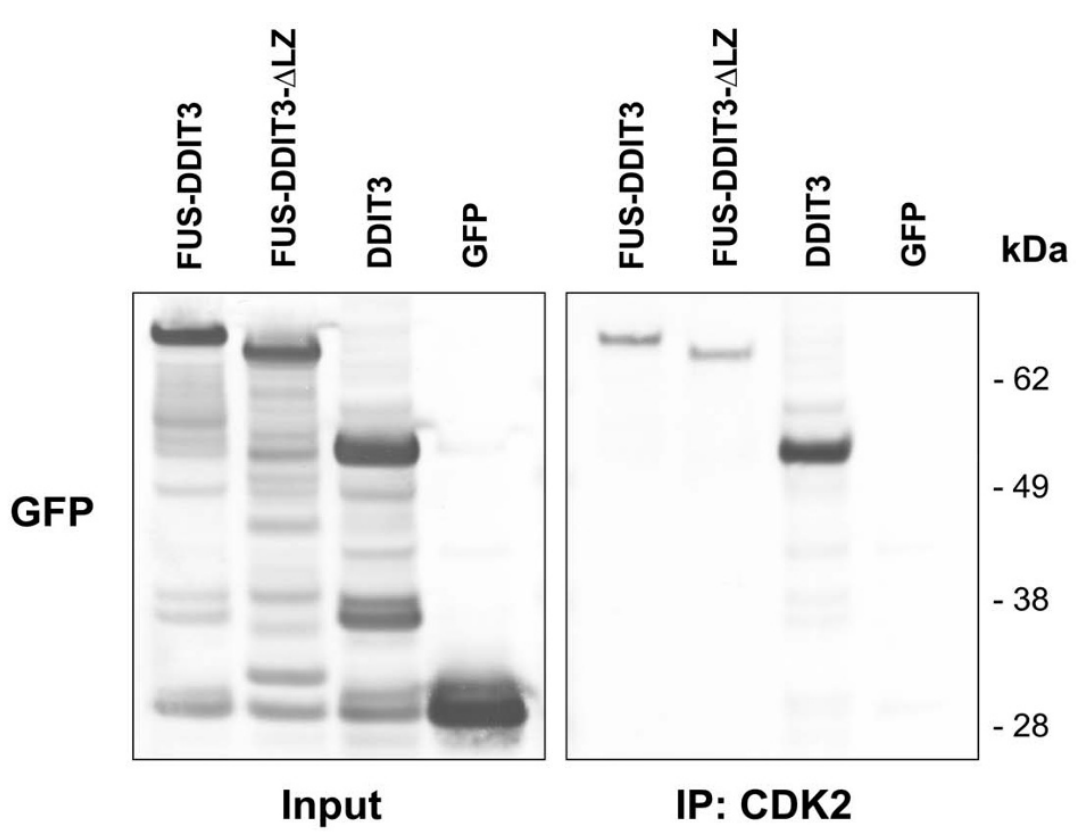

(c)

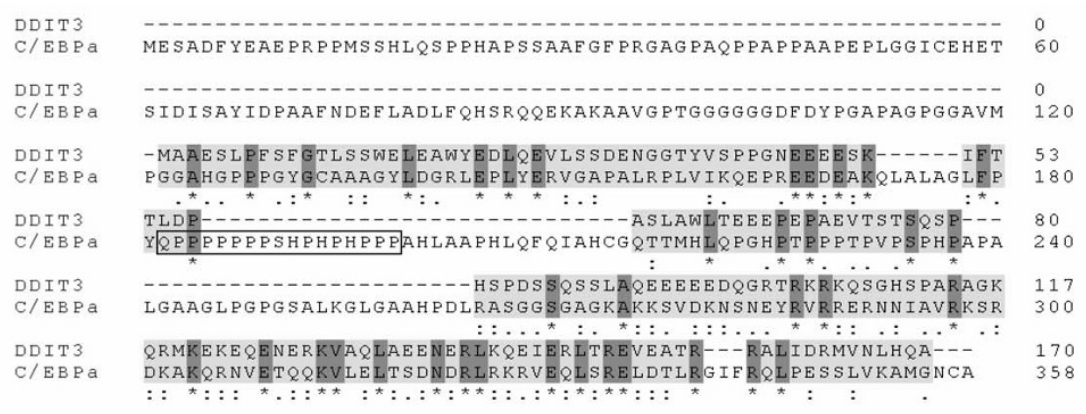

Figure 2

Co-immunoprecipitation of DDIT3 and CDK2. (a) HTI 080 cells were co-transfected with the indicated GFP constructs and CDK2-DsRed I. Proteins in cell lysates were further immunoprecipitated with anti GFP serum and recombinant CDK2DsRed I protein was detected by western blot using CDK2 antibodies. Input shows proteins before immunoprecipitation of cell lysates while IP shows proteins present in immunoprecipitates. (b) HTI080 cells were transiently transfected with the indicated GFP constructs and cellular proteins were immunoprecipitated with CDK2 antibodies. Recombinant proteins in samples were detected with anti GFP serum. The most intense bands in the input show the correct size of the GFP-tagged protein products while additional unspecific bands were also reacting with the GFP antisera in these samples. (c) ClustalW alignment of DDIT3 and C/EBP $\alpha$ amino acid sequences. A region shown to bind CDK2 in C/EBP $\alpha$ is boxed. Letters indicate standard amino acid abbreviations. Amino acids with related physicochemical characteristics are displayed below the sequences as: '*' (identical residue), ':' (highly similar residue), '.' (weakly similar residue). The leucine zipper region is seen towards the C-terminal in both proteins. 


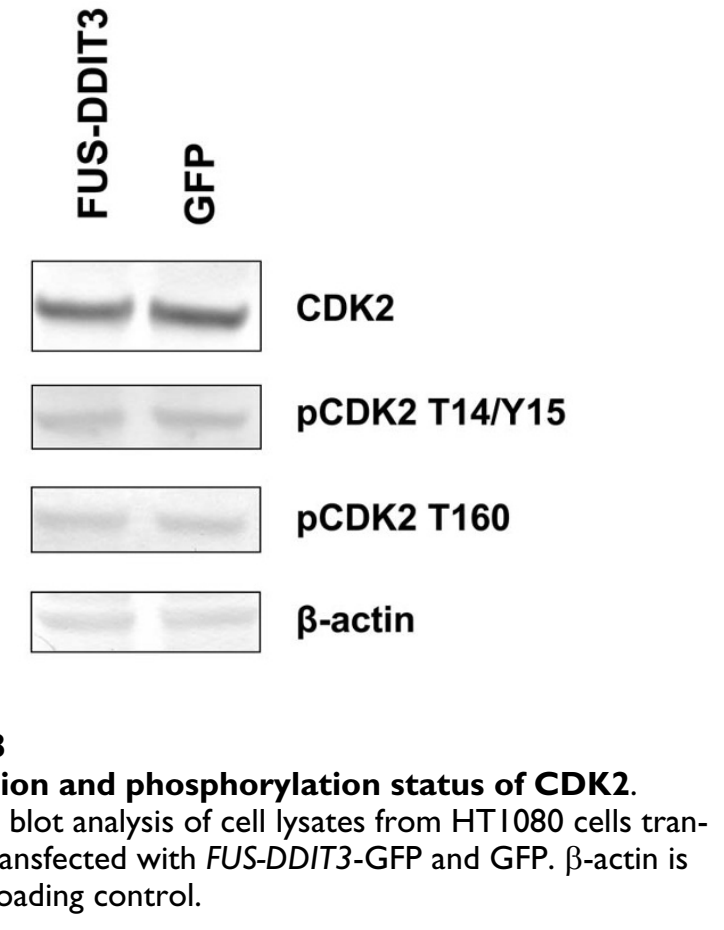

Figure 3

Expression and phosphorylation status of CDK2.

Western blot analysis of cell lysates from HTI080 cells transiently transfected with FUS-DDIT3-GFP and GFP. $\beta$-actin is used as loading control.

\section{CDK2 expression, phosphorylation status and turnover is not changed by FUS-DDIT3}

The CDK2 expression in HT1080 cells after 42 hours of transfection with FUS-DDIT3-GFP or GFP was investigated by western blot analysis (Figure 3 ). In addition, the phosphorylation status of CDK2 was analyzed using CDK2 antibodies targeting either the two inhibitory phosphorylations at threonine 14 and tyrosine 15 or the activating phosphorylation at threonine 160 (Figure 3). No apparent difference in CDK2 phosphorylation between cells expressing FUS-DDIT3-GFP or GFP was detected and the amount of CDK2 between the two transfected cell populations was equivalent (Figure 3 ). The CDK2 protein half-life did not differ between stably transfected FUSDDIT3 cells and HT1080 control cells upon a six hour cycloheximide chase assay (not shown).

\section{CDK2 protein binding affinity is altered in FUS-DDIT3 and DDIT3 expressing cells}

HT1080 cells were transfected with either of FUS-DDIT3, DDIT3 or GFP and CDK2 was immunoprecipitated from lysates with CDK2 specific antibodies (Figure 4). Precipitates were analyzed by SDS polyacrylamide gel electrophoresis and stained with Coomassie Blue. Several bands were present in the FUS-DDIT3 and DDIT3 precipitates that were absent in precipitates from GFP expressing cells (Figure 4). Bands from three regions were cut as indicated in the figure and analyzed by LC-MS/MS. The cytoskeletal cross-linker plectin (Figure 4, region 1), the actin motorprotein myosin (Figure 4, region 2 ) and the intermediary

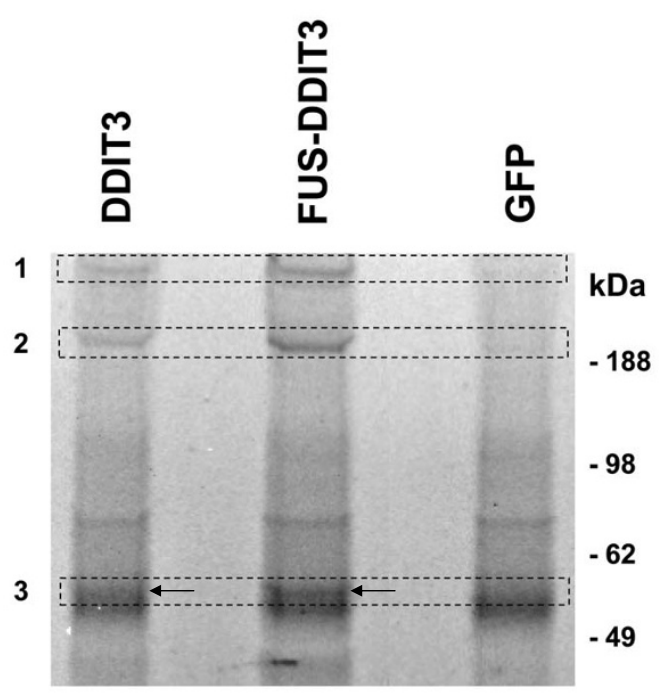

IP: CDK2

\section{Figure 4}

SYPRO stained gel of co-immunoprecipitated proteins in extracts from HT 1080 cells transiently transfected with either of DDIT3-GFP, FUS-DDIT3-GFP or GFP constructs. CDK2 antibodies were used for immunoprecipitations. Boxes indicate regions of the gel analyzed by mass spectrometry. Three proteins were enriched in immunoprecipitates from DDIT3 and FUS-DDIT3 expressing cells (region I: plectin, region 2: myosin, region 3: vimentin). Arrows indicate the differentially immunoprecipitated vimentin bands present in lanes of DDIT3 and FUS-DDIT3 cells but absent in that of GFP cells. These bands migrate slightly slower than the thicker bands inside the same region. The thicker bands were identified as rabbit heavy chain immunoglobulin molecules and these originate from the antibodies used for immunoprecipitation of CDK2 and are present in all three lanes.

filament protein vimentin (Figure 4, region 3) were enriched in immunoprecipitates of FUS-DDIT3 and DDIT3 cells. The thicker bands with similar abundance in all three lanes in region 3 were identified by mass spectrometry as rabbit heavy chain immunoglobulin molecules.

\section{Discussion}

In the present study, we used immunofluorescence microscopy to investigate putative colocalization between FUSDDIT3 and cyclins/cyclin-dependent kinases involved in G1 cell cycle control. CDK2 and the CDK2 binding cyclin E were found to change their localization in FUS-DDIT3 expressing cells to a pattern that was identical to the FUSDDIT3 nuclear granules reported earlier [24]. This suggests that CDK2 and cyclin E are translocated to nuclear structures defined by the FUS-DDIT3 protein. As the 
DDIT3 part is considered a DNA-binding transcription factor [27], we speculate that the FUS-DDIT3 defined structures may contain active chromatin and mRNA at different stages of processing. Thus, the accumulation of CDK2 and cyclin E to such nuclear regions may result in changed phosphorylation patterns and regulation of substrates present at these foci.

DDIT3 is able to form DNA-binding heterodimers with C/ EBP $\alpha$ through its leucine zipper region [14] and C/EBP $\alpha$ is reported to bind CDK2 and inhibit its kinase activity [26]. Therefore, it is possible that the binding between DDIT3 and CDK2 is mediated through C/EBP $\alpha$ or another C/EBP protein. However, we here show that a DDIT3 mutant lacking the leucine zipper domain binds CDK2, which implies a binding between DDIT3 and CDK2 that is independent of other C/EBP proteins. DDIT3 and C/EBP $\alpha$ contain several regions with sequence similarities but the proline-histidine rich part reported to bind CDK2 in C/EBP $\alpha$ [26] (Figure 2c) is not present in DDIT3. This suggests that DDIT3 binds CDK2 through a different mechanism than C/EBP $\alpha$.

Analyses of GFP-immunoprecipitates failed to detect cyclin E in these samples. The absence of cyclin E in CDK2 immunoprecipitates bears resemblance to previous reports showing that $\mathrm{C} / \mathrm{EBP} \alpha$ disrupts $\mathrm{CDK} 2 /$ cyclin complexes leading to growth arrest [26]. Hence, it is possible that the DDIT3 bound to CDK2 disrupts CDK2/cyclin E complexes in similar way as $\mathrm{C} / \mathrm{EBP} \alpha$. Consequently, since cyclin $\mathrm{E}$ is a major regulator of CDK2 kinase activity in G1, the DDIT3 binding may alter CDK2 activity in DDIT3 and FUS-DDIT3 expressing cells. We did however not detect a change in phosphorylation status of CDK2 in FUS-DDIT3 expressing cells compared to control cells. To further analyze the functional effects of DDIT3/FUS-DDIT3 binding to CDK2, we immunoprecipitated CDK2 in cells transiently transfected with FUS-DDIT3, DDIT3 and GFP constructs. Analysis of the precipitates revealed enhanced binding of CDK2 to components of the cytoskeleton in cells expressing FUS-DDIT3 and DDIT3. An increased affinity for cytoskeletal components and crosstalk between cell cycle proteins and cytoskeletal regulatory proteins could lead to changes in cytoskeleton structure [28].

\section{Conclusions}

In conclusion, we show that CDK2 is translocated to nuclear structures defined by the FUS-DDIT3 oncoprotein and that it binds the DDIT3 part of the chimera. Cyclin E is also recruited to FUS-DDIT3 nuclear structures but can not be found in CDK2-containing DDIT3/FUS-DDIT3 precipitates. The interaction of FUS-DDIT3 and DDIT3 with CDK2 appears to alter the binding affinity of CDK2, possibly leading to changed phosphorylation patterns and regulation of cytoskeletal or other proteins. Many of the observed biological effects of DDIT3 may involve the interaction with CDK2.

\section{Methods \\ Cell culture and transfection}

Human HT1080 fibrosarcoma cells were grown in RPMI 1640 medium (Sigma-Aldrich) supplied with $10 \%$ fetal bovine serum, penicillin $(50 \mathrm{U} / \mathrm{ml})$ and streptomycin $(50$ $\mu \mathrm{g} / \mathrm{ml})$ at $37^{\circ} \mathrm{C}$ in $5 \% \mathrm{CO}_{2}$. Cells were transiently transfected at 50-70\% confluence using the FuGENE 6 transfection reagent (Roche) with a 3:1 FuGENE:DNA ratio, according to the instructions supplied by the manufacturer.

\section{Immunofluorescence}

Cells were fixed 24 hours after transfection in 3.7\% formaldehyde in PBS for 10 min, rinsed in PBS and stained with rabbit polyclonal antibodies for CDK2 (C5223, Sigma-Aldrich), CDK4 (C8218, Sigma-Aldrich), cyclin D1 (M7155, Dako) or cyclin E (C4976, Sigma-Aldrich) that were detected using Cy3 conjugated secondary antibodies (Fluorolink, Amersham Biosciences). Finally, slides were mounted using DAPI/DABCO solution (Sigma-Aldrich), incubated over night at room temperature and then imaged using a Zeiss LSM510 META confocal microscope system.

\section{Expression vectors}

For cloning of CDK2 the following primers were used: CDK2 forward GATCTCGAGCCACCATGGAGAACTTCCAAAAG, CDK2 reverse CAAGGATCCCGGAGTCGAAGATGGGGTACT. The CDK2 fragment was cloned into the pDsRed1-N1 vector (Clontech Laboratories) in frame with the DsRed 1 sequence. All constructs were sequenced to exclude mutant clones. FUS-DDIT3-GFP, DDIT3-GFP and FUSA-GFP were previously described $[24,29]$. The FUS-DDIT3 LZ-GFP construct, expressing a protein lacking the 38 most $\mathrm{C}$-terminal amino acids (including the leucine zipper region), is described elsewhere [30]. Stably transfected FUS-DDIT3 cells has been described previously [31].

\section{Co-immunoprecipitation}

HT1080 cells were co-transfected with CDK2-DsRed and/ or FUS-DDIT3-GFP/FUS-DDIT3- $\triangle$ LZ/DDIT3-GFP/FUSAGFP/GFP constructs at $50-70 \%$ confluence. Twenty-four hours post transfection, cells were washed in PBS, scraped and lysed in NP-40 buffer (150 mM NaCl, 1\% NP-40, 50 $\mathrm{mM}$ Tris $\mathrm{pH}$ 8.0) containing protease inhibitor (Complete Mini, Roche). Debris was removed by centrifugation at $14,000 \mathrm{~g}$ and supernatants were used for co-immunoprecipitations. Two microgram of antibodies specific for GFP (8372-2, BD Pharmingen) or CDK2 (C5223, SigmaAldrich) were prebound to protein A agarose beads (Mill- 
ipore) blocked with 5\% bovine serum albumin in NP-40 buffer overnight. Cell lysates were incubated with antibody-beads mixtures for $4 \mathrm{~h}$ at room temperature. Beads were washed 5 times with NP-40 buffer and proteins were released from beads by addition of $4 \times$ NuPAGE LDS sample buffer (Invitrogen) and incubation at $95^{\circ} \mathrm{C}$. Supernatants were subsequently analyzed with western blot or with SYPRO Ruby gel staining (Sigma-Aldrich) according to the instructions supplied by the manufacturer. SYPRO stained gels were analyzed by liquid chromatographymass spectrometry (LC-MS/MS) at the Proteomics Core Facility at University of Gothenburg. For experiments with phospho-CDK2 antibodies (see below), transfected cells were lysed in RIPA buffer (50 mM Tris-HCl pH 7.4, 150 $\mathrm{mM} \mathrm{NaCl}, 1 \mathrm{mM}$ EDTA, 1\% Triton $\mathrm{x}-100,1 \%$ Sodium deoxycholate, $0.1 \%$ SDS) containing protease inhibitors and phosphatase inhibitors ( $\mathrm{NaF} 10 \mathrm{mM}, \mathrm{Na}_{3} \mathrm{VO}_{4} 1 \mathrm{mM}$ ).

\section{Western Blot Analysis}

Proteins were separated using NuPAGE 4-12\% Bis-Tris gels (Invitrogen), blotted onto PVDF membranes (Millipore) and probed with the following primary antibodies: CDK2 (sc-6248, Santa Cruz Biotechnology), p-CDK2 Thr14/Tyr15 (Santa Cruz Biotechnology), p-CDK2 Thr160 (Cell Signaling), cyclin E (C4976, Sigma-Aldrich), $\beta$-actin (mAbcam8226, Abcam) and GFP (8371-2, BD Pharmingen). Primary antibodies were detected with alkaline phosphatase conjugated secondary antibodies (Dako) and bands were visualized with BCIP/NBT Alkaline Phosphatase Substrate (Sigma-Aldrich).

\section{ClustalW alignment}

The amino acid sequences of DDIT3 and C/EBP $\alpha$ were aligned using the ClustalW algorithm available at the Universal protein resource (UniProt) [32].

\section{Abbreviations}

CDK2: cyclin-dependent kinase 2; C/EBP: CCAAT/ enhancer binding protein; CHOP: C/EBP homologous protein; DDIT3: DNA damage-inducible transcript 3; FUS: fusion; GFP: green fluorescent protein; LC-MS/MS: liquid chromatography mass spectrometry; MLS/RCLS: myxoid/ round-cell liposarcoma; TLS: translocated in liposarcoma.

\section{Authors' contributions}

$\mathrm{PA}$ conceived of the study. CB, MKA and PA designed the experiments. $\mathrm{CB}$ and MKA performed research. $\mathrm{CB}, \mathrm{MKA}$ and $P \AA$ wrote the manuscript. All authors read and approved the final version of the manuscript.

\section{Acknowledgements}

We thank Anita Olofsson and Farzaneh Alemirad for technical assistance. We acknowledge the Proteomics Core Facility at University of Gothenburg for mass spectrometric analyses. This work was supported by grants from the Swedish Cancer Society, Assar Gabrielssons Research Foundation and the Johan Jansson Foundation for Cancer Research.

\section{References}

I. Ramji DP, Foka P: CCAAT/enhancer-binding proteins: structure, function and regulation. Biochem $J$ 2002, 365(Pt 3):56I-575.

2. Fornace AJ Jr, Nebert DW, Hollander MC, Luethy JD, Papathanasiou M, Fargnoli J, Holbrook NJ: Mammalian genes coordinately regulated by growth arrest signals and DNA-damaging agents. Mol Cell Biol 1989, 9( I 0):4196-4203.

3. Carlson SG, Fawcett TW, Bartlett JD, Bernier M, Holbrook NJ: Regulation of the C/EBP-related gene gadd 153 by glucose deprivation. Mol Cell Biol 1993, I3(8):4736-4744.

4. Sylvester SL, ap Rhys CM, Luethy-Martindale JD, Holbrook NJ: Induction of GADDI 53, a CCAAT/enhancer-binding protein (C/EBP)-related gene, during the acute phase response in rats. Evidence for the involvement of C/EBPs in regulating its expression. I Biol Chem 1994, 269(3I):20119-20125.

5. Guyton KZ, Xu Q, Holbrook NJ: Induction of the mammalian stress response gene GADDI 53 by oxidative stress: role of AP-I element. Biochem J 1996, 3 I 4(Pt 2):547-554.

6. Wang XZ, Lawson B, Brewer JW, Zinszner H, Sanjay A, Mi LJ, Boorstein R, Kreibich G, Hendershot LM, Ron D: Signals from the stressed endoplasmic reticulum induce C/EBP-homologous protein (CHOPIGADD I 53). Mol Cell Biol I996, I 6(8):4273-4280.

7. Barone MV, Crozat A, Tabaee A, Philipson L, Ron D: CHOP (GADDI53) and its oncogenic variant, TLS-CHOP, have opposing effects on the induction of GI/S arrest. Genes Dev 1994, 8(4):453-464.

8. Zinszner $\mathrm{H}$, Kuroda $M$, Wang $X$, Batchvarova $N$, Lightfoot $R T$, Remotti H, Stevens JL, Ron D: CHOP is implicated in programmed cell death in response to impaired function of the endoplasmic reticulum. Genes Dev 1998, I 2(7):982-995.

9. Song B, Scheuner D, Ron D, Pennathur S, Kaufman RJ: Chop deletion reduces oxidative stress, improves beta cell function, and promotes cell survival in multiple mouse models of diabetes. J Clin Invest 2008, I I 8( I 0):3378-3389.

10. Oyadomari S, Mori M: Roles of CHOP/GADDI53 in endoplasmic reticulum stress. Cell Death Differ 2004, I I (4):38I-389.

II. Batchvarova N, Wang XZ, Ron D: Inhibition of adipogenesis by the stress-induced protein CHOP (Gadd I 53). EMBO J 1995 , I4( I9):4654-466I.

12. Coutts M, Cui K, Davis KL, Keutzer JC, Sytkowski AJ: Regulated expression and functional role of the transcription factor CHOP (GADDI53) in erythroid growth and differentiation. Blood 1999, 93(1 0):3369-3378.

13. Pereira RC, Delany AM, Canalis E: CCAAT/enhancer binding protein homologous protein (DDIT3) induces osteoblastic cell differentiation. Endocrinology 2004, I45(4): | $952-1960$.

14. Ron D, Habener JF: CHOP, a novel developmentally regulated nuclear protein that dimerizes with transcription factors $\mathrm{Cl}$ EBP and LAP and functions as a dominant-negative inhibitor of gene transcription. Genes Dev 1992, 6(3):439-453.

15. Chen BP, Wolfgang CD, Hai T: Analysis of ATF3, a transcription factor induced by physiological stresses and modulated by gadd I 53/Chop I 0. Mol Cell Biol 1996, I 6(3): I I 57- I I 68.

16. Ubeda $M$, Vallejo $M$, Habener JF: CHOP enhancement of gene transcription by interactions with Jun/Fos AP-I complex proteins. Mol Cell Biol I999, I 9( I I):7589-7599.

17. Riggi N, Cironi L, Provero P, Suva ML, Stehle JC, Baumer K, Guillou L, Stamenkovic I: Expression of the FUS-CHOP fusion protein in primary mesenchymal progenitor cells gives rise to a model of myxoid liposarcoma. Cancer Res 2006, 66(14):7016-7023.

18. Perez-Losada J, Pintado B, Gutierrez-Adan A, Flores T, BanaresGonzalez B, del Campo JC, Martin-Martin JF, Battaner E, SanchezGarcia I: The chimeric FUS/TLS-CHOP fusion protein specifically induces liposarcomas in transgenic mice. Oncogene 2000, I9(20):24I 3-2422.

19. Crozat A, Åman P, Mandahl N, Ron D: Fusion of CHOP to a novel RNA-binding protein in human myxoid liposarcoma. Nature 1993, 363(6430):640-644.

20. Rabbitts TH, Forster A, Larson R, Nathan P: Fusion of the dominant negative transcription regulator $\mathrm{CHOP}$ with a novel gene FUS by translocation $t(12 ; 16)$ in malignant liposarcoma. Nat Genet 1993, 4(2): I75-I80. 
2I. Panagopoulos I, Hoglund M, Mertens F, Mandahl N, Mitelman F, Aman $P$ : Fusion of the EWS and CHOP genes in myxoid liposarcoma. Oncogene 1996, I 2(3):489-494.

22. Sanchez-Garcia I, Rabbitts TH: Transcriptional activation by TALI and FUS-CHOP proteins expressed in acute malignancies as a result of chromosomal abnormalities. Proc Natl Acad Sci USA 1994, 9 I(I 7):7869-7873.

23. Goransson $M$, Wedin M, Aman P: Temperature-dependent localization of TLS-CHOP to splicing factor compartments. Exp Cell Res 2002, 278(2): I25-I32.

24. Thelin-Jarnum S, Goransson M, Burguete AS, Olofsson A, Aman P: The myxoid liposarcoma specific TLS-CHOP fusion protein localizes to nuclear structures distinct from PML nuclear bodies. Int J Cancer 2002, 97(4):446-450.

25. Olofsson A, Willen H, Goransson M, Engstrom K, Meis-Kindblom JM, Stenman G, Kindblom LG, Aman P: Abnormal expression of cell cycle regulators in FUS-CHOP carrying liposarcomas. Int J Oncol 2004, 25(5): I349-I355.

26. Wang $H$, lakova $P$, Wilde $M$, Welm A, Goode T, Roesler WJ, Timchenko NA: C/EBPalpha arrests cell proliferation through direct inhibition of Cdk2 and Cdk4. Mol Cell 200I, 8(4):8I7-828.

27. Ubeda M, Wang XZ, Zinszner H, Wu I, Habener JF, Ron D: Stressinduced binding of the transcriptional factor CHOP to novel DNA control element. Mol Cell Biol 1996, I 6(4): | 479- | 489.

28. Besson A, Assoian RK, Roberts JM: Regulation of the cytoskeleton: an oncogenic function for CDK inhibitors? Nat Rev Cancer 2004, 4(I 2):948-955.

29. Andersson MK, Stahlberg A, Arvidsson Y, Olofsson A, Semb H, Stenman G, Nilsson O, Aman P: The multifunctional FUS, EWS and TAFI5 proto-oncoproteins show cell type-specific expression patterns and involvement in cell spreading and stress response. BMC Cell Biol 2008, 9:37.

30. Goransson M, Andersson MK, Forni C, Stahlberg A, Andersson C Olofsson A, Mantovani R, Aman P: The myxoid liposarcoma FUS-DDIT3 fusion oncoprotein deregulates NF-kappaB target genes by interaction with NFKBIZ. Oncogene 2009, 28(2):270-278.

31. Goransson M, Elias E, Stahlberg A, Olofsson A, Andersson C, Aman $P$ : Myxoid liposarcoma FUS-DDIT3 fusion oncogene induces C/EBP beta-mediated interleukin 6 expression. Int J Cancer 2005, I I 5(4):556-560.

32. The universal protein resource (UniProt). Nucleic Acids Res 2008:D190-195.
Publish with Bio Med Central and every scientist can read your work free of charge

"BioMed Central will be the most significant development for disseminating the results of biomedical research in our lifetime. "

Sir Paul Nurse, Cancer Research UK

Your research papers will be:

- available free of charge to the entire biomedical community

- peer reviewed and published immediately upon acceptance

- cited in PubMed and archived on PubMed Central

- yours - you keep the copyright
BioMedcentral 